\title{
Berufliche Rehabilitation: First Place, then Train - Kontra
}

\author{
Vocational Rehabilitation: First Place, then Train - Contra
}
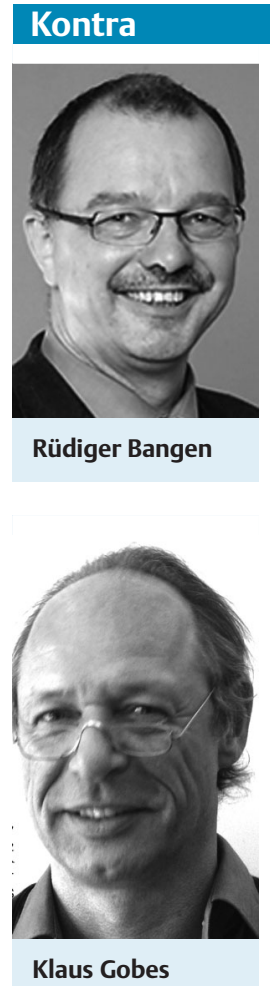

Klaus Gobes
Rüdiger Bangen

Wir möchten in unserer Ausführung bewusst und möglichst auf die Unterstützung von Studien verzichten. Wir bemühen unsere Erfahrungswerte sowie unseren (geglaubten) „unabhängigen“ therapeutischen Verstand, dem wir auch nach Jahren Vertrauen schenken ohne die Angst, zu kristallin geworden zu sein. In Anbetracht der nur noch kurzfristigen Behandlungszeiträume von doch schwerwiegenden Erkrankungen in den psychiatrischen Kliniken müsste es richtigerweise heißen, erst eine Behandlungsgrundlage schaffen, dann trainieren und stabilisieren und dann erst erfolgreich platzieren und die Behandlungsgrundlage beibehalten. Wenn man die evidenzbasierten Elaborate der wissenschaftlichen Eliten zu Rate zieht, so erhalten wir immer Bezüge zum amerikanischen Weg, wobei wir hier im alten Europa wissen müssten, dass wir die eigentliche Geschichte der Sozialpsychiatrie und Arbeitstherapie geschrieben haben [1-3] bzw. noch am Schreiben sind. Was wir nicht in Abrede stellen wollen ist, dass alle Hilfeleistungen willkommen sind sowie einen Nutzen und ihre Berechtigung haben, wenn sie durch die Betroffenen akzeptiert werden. An dieser Stelle möchten wir unser Schulsystem kurz in den Fokus rücken. Die Reformen der letzten 35-40 Jahre haben nicht die gewünschten Erfolge gebracht, wir erleben eher eine Nivellierung zum statistischen Mittel, möglicherweise haben die Fachkenntnisse in einzelnen Teilberei- chen zugenommen, aber die Erkenntnis über das Zusammenwirken aller Fächer, die Persönlichkeitsreife und die praktische Intelligenzentwicklung bleiben auf der Strecke. Was nutzt PISA, wenn ich noch nicht einmal einen Fahrkartenautomat bedienen kann respektive mich Mami zum Studieren fahren muss [4, 5] Aber was soll es wirklich helfen, wenn wir ohne Grundlage und ohne Trainingssicherheit bereits in das Rennen geschickt werden? Jeder Sportler oder auch Schachspieler trainiert vor einem Wettbewerb, für jede Klassenarbeit üben wir zuerst, bevor wir uns dem Stress der realen Situation aussetzen - Schritt für Schritt, und diese Schritte sollten sicher sein.

Ein persönlicher Begleiter soll es vor Ort richten. Natürlich sollte er im Idealfall eine umfassende, projektbezogene Ausbildung nachweisen. Uns sind im deutschen Betreuungswesen die Schwierigkeiten bewusst: Entsprechendes Personal zu finden ist nicht einfach, weiterhin ist fraglich, inwiefern ein Betrieb auf Dauer einen „schnellen Brüter“ in seinem System etablieren und akzeptieren kann, wie weit dessen Befugnisse reichen und welche Sonderstellung ein Betriebsangehöriger einnimmt, der einen solchen Schattenmann an seiner Seite hat. Wir haben auch hier schon Beispiele, die im Rahmen von Beschäftigungsprojekten im Sinne von Selbsthilfefirmen funktionieren. Aber ist das echte Integration? Häufig sieht die Situation einer Fachabteilung im Betrieb so aus, dass ein spezifischer Personenkreis besonders betreut und trainiert wird, um das tagtägliche Arbeitsaufkommen kompensieren zu können. Wirklich qualifizierte Jobs entstehen dabei nicht, knüpfen diese doch an unsere kulturelle Tradition beruflicher Ausbildung eines dualen Bildungswesens an, deren Ergebnisse hiesige Arbeitgeber die notwendige Sicherheit für ihr unternehmerisches Tun garantieren. Selbsthilfefirmen erhalten ihre Existenzgrundlage in dem Gros der Fälle durch Subventionen. Platzierte und dann trainierte psychisch kranke Arbeitnehmer riskieren poor jobs, also weiterhin prekäre Arbeitssituationen, mit denen die Gefahr erneuter psychischer Erkrankungen einhergeht.

Wir sollten die höchstrichterlichen Rechtsprechungen [6] nicht ganz vergessen: Psychische Erkrankungen entstehen dann, wenn deren Symptome sich der willentlichen Beeinflussung des Betroffenen entziehen. RPK - Rehabilitation Psychisch Kranker - ist ein Einrichtungstyp, der 1987 entstanden ist und heute auf der RPK-Empfehlungsvereinbarung vom 29. September 2005 über die Zusammenarbeit der Krankenversicherungsträger und der Rentenversicherungsträger sowie der Bundesagentur für Arbeit bei der Gewährung von Leistungen zur Teilhabe in Rehabilitationseinrichtungen für psychisch kranke und behinderte Menschen basiert. Hier möchten wir betonen, dass die Einrichtungen, die die Empfehlungsvereinbarung ernsthaft und nachhaltig realisiert haben, das derzeit optimierte rehabilitativ arbeitende Therapeutikum darstellen. Wir halten ein Becken voll von gut ausgebildeten Fachkräften bereit (Medizin, Psychologie, Sozialpädagogik, Fachkrankenpflege, Physiotherapie, Hauswirtschaft u.a.) und bieten eine Auswahl von Förderstätten mit einem gestaffelten Anforderungsprofil an, sodass das Bildungsgefüge unserer Gesellschaft Berücksichtigung finden kann. Wir haben die Zeit, den Menschen mit seiner Individualität kennenzulernen und so maßgeschneiderte Förderpläne zu gestalten. Von der Akutbehandlung über die Persönlichkeitsnachreife hin zu einer angepassten Belastungserprobung in einer realen Arbeitssituation ohne direkten Schattenmann, jedoch mit einem dichten Netz an Therapeutika der beschriebenen Berufsgruppen wird von uns alles ermöglicht. Im Rahmen der Empfehlungsvereinbarung ist es auch gelungen, alle Leistungsträger an den Tisch zu bekommen, wobei ein reibungsarmer Ablauf allerdings immer noch nicht wirklich realisiert werden konnte. 
Eine Bestätigung unseres ganzheitlichen, trainierenden Ansatzes findet sich auch in der neuen Entwicklungsrichtung der Verhaltenstherapie, der „fünften Welle“ [7]. Diese führt fachübergreifend die therapeutischen Ansätze zusammen, die den Betroffenen wieder selbststeuerungsfähiges Handeln ermöglichen sollen. Wir schreiben also auch in diesem Zusammenhang bereits mit am großen wissenschaftlichen Buch der Erkenntnis. Dieses kennt offensichtlich auch die OECD [8], welche bereits im Jahr 2010 ihren Mitgliedern nahegelegt hat, medizinisches Therapeutikum und berufliche Förderung gleichsam für eine erfolgreiche Arbeitsintegration zu verbinden. MBOR (medizinisch-berufliche Orientierung in der Rehabilitation): die Zukunft für den Erfolg der Rehabilitation ist ebenfalls auch Garant für die beste Wirtschaftlichkeit erbrachter Leistungen.

Ebenfalls gilt es zu klären, auf welche Art und Weise sich der Personenkreis der Betroffenen zusammensetzt, dem wir Hilfsmaßnahmen angedeihen lassen. In der Majorität der Fälle handelt sich es um Menschen mit Diagnosen aus den ICD10-Bereichen F2, F3, F4 und F 6. Im Durchschnitt liegen vor Aufnahme in eine RPK drei stationäre Aufenthalte in psychiatrischen Krankenhäusern und nur die Wenigsten $(<10 \%)$ verfügen noch über ein aktuelles Arbeitsverhältnis oder haben überhaupt schon erfolgreich am allgemeinen Arbeitsmarkt partizipiert. Die Aufnahme erfolgt in der Regel direkt nach einer Krankenhausbehandlung.
Hier einige Positionen von Betroffenen, direkt aus der Praxis: „Ich habe jetzt 3 Monate, ja! solange, gebraucht um einzusehen, dass ich die ganze Sache nur für mich mache.“ (Herr G.). „Nachdem ich einen erneuten Studienversuch, eine Anwaltskanzlei und diverse Handwerksbetriebe testen konnte, weiß ich, wo mich mein Weg hinführen wird." (Herr S.). „Die unterschiedlichen betrieblichen Praktika im Rahmen meiner Belastungserprobung haben mir mein Leistungsvermögen widergespiegelt und mich in meinem Entschluss bestärkt, mein Studium zu Ende bringen zu wollen.“ (Frau M.) Herr G. befindet sich noch aktuell in seiner Maßnahme. Herr S. hat die Aufnahmeprüfung zur Finanzbeamtenlaufbahn realisiert. Frau M. musste ihr Studium nach 2 erfolgreichen Semestern doch abbrechen, da ihre Zweiterkrankung, eine Epilepsie, keine Alternative zuließ.

In Anbetracht der vielfältigen Probleme in unserem Gesundheitssystem tut man gut daran, mit einem verlässlichen Produkt zu punkten, welches Optimierungsoptionen einschließt. Es ist müßig, immer wieder auf ein neues Pferd zu setzen, das noch nicht wirklich zugeritten ist. Es geht nicht um Stagnation, sondern um einen vernunftbetonten Umgang mit Ressourcen.

Sie haben eine eigene Meinung zu diesem

Thema? Dann schreiben Sie uns an:

psychiat-praxis@thieme.de!

\section{Literatur}

1 Paetz A. Die Kolonisierung der Geisteskranken in Verbindung mit dem Offen-Thür-System. Berlin: Verlag Julius Springer; 1893

2 Simon H. Aktivere Krankenbehandlung in der Irrenanstalt. Berlin: Walter de Gruyter \& Co; 1929

3 Klee E. Die armen Irren. Das Schicksal der seelisch Kranken. Düsseldorf: Patmos-Verlag; 1972

4 Spreckelstein T. Warum ist G8 gescheitert. Frankfurter Allgemeine Zeitung Feuilleton 28.02.2014

5 Kaube J. Vom Unsinn ewiger Bildungsreformen. Frankfurter Allgemeine Zeitung Feuilleton 11.05.2014

6 Bundessozialgericht. 9RV 556/71 vom 16.05 .1972

7 Fiedler $P$. Neue Welle der Verhaltenstherapie. Nervenheilkunde 2014; 4: 227-232

8 OECD. Maladie, invalidité et travail; Synthèse des résultats dans les pays de l'OCDE. OECD; 2010

Korrespondenzadresse

Dipl.-Psych. Klaus Gobes

Psychologischer Psychotherapeut

REAS-RPK Modau

Kirchstraße 39

64372 Ober-Ramstadt/Nieder-Modau

kgobes@reas.de

Bibliografie

DOI http://dx.doi.org/

10.1055/s-0034-1370233

Psychiat Prax 2014; 41: 295-296

(c) Georg Thieme Verlag KG

Stuttgart · New York

ISSN 0303-4259 